\title{
Communication
}

\section{Graphene Near-Degenerate Four-Wave Mixing for Phase Characterization of Broadband Pulses in Ultrafast Microscopy}

Richard Ciesielski, Alberto Comin, Matthias Handloser, Kevin Donkers, Giovanni Piredda, Antonio Lombardo, Andrea C Ferrari, and Achim Hartschuh

Nano Lett., Just Accepted Manuscript • DOI: 10.1021/acs.nanolett.5b00893 • Publication Date (Web): 29 Jun 2015

Downloaded from http://pubs.acs.org on July 2, 2015

\section{Just Accepted}

"Just Accepted" manuscripts have been peer-reviewed and accepted for publication. They are posted online prior to technical editing, formatting for publication and author proofing. The American Chemical Society provides "Just Accepted" as a free service to the research community to expedite the dissemination of scientific material as soon as possible after acceptance. "Just Accepted" manuscripts appear in full in PDF format accompanied by an HTML abstract. "Just Accepted" manuscripts have been fully peer reviewed, but should not be considered the official version of record. They are accessible to all readers and citable by the Digital Object Identifier (DOI®). "Just Accepted" is an optional service offered to authors. Therefore, the "Just Accepted" Web site may not include all articles that will be published in the journal. After a manuscript is technically edited and formatted, it will be removed from the "Just Accepted" Web site and published as an ASAP article. Note that technical editing may introduce minor changes to the manuscript text and/or graphics which could affect content, and all legal disclaimers and ethical guidelines that apply to the journal pertain. ACS cannot be held responsible for errors or consequences arising from the use of information contained in these "Just Accepted" manuscripts. 


\title{
Graphene Near-Degenerate Four-Wave Mixing
} for Phase Characterization of Broadband Pulses in Ultrafast Microscopy

\author{
Richard Ciesielski ${ }^{1},{ }^{\dagger}$ Alberto Comin ${ }^{1},{ }^{\dagger}$ Matthias Handloser ${ }^{1},{ }^{\dagger}$ Kevin \\ Donkers $^{1},{ }^{\dagger}$ Giovanni Piredda ${ }^{2},{ }^{\dagger}$ Antonio Lombardo ${ }^{3},{ }^{\dagger}$ Andrea C. Ferrari ${ }^{3}{ }^{\dagger}$ \\ and Achim Hartschuh ${ }^{1 * \dagger}$ \\ ${ }^{1}$ Department Chemie and CeNS, Ludwig Maximilians Universität München, Butenandtstr. 5-13, \\ 81377 Munich, Germany, ${ }^{2}$ Josef Ressel Zentrum, Fachhochschule Vorarlberg, Hochschulstr. 1, \\ 6850 Dornbirn, Austria, and ${ }^{3}$ Cambridge Graphene Centre, University of Cambridge, $9 \mathrm{JJ}$ \\ Thomson Avenue, Cambridge CB30FA, UK \\ E-mail: achim.hartschuh@cup.uni-muenchen.de
}

\begin{abstract}
We investigate near-degenerate four-wave mixing in graphene using femtosecond laser pulse shaping microscopy. Intense near-degenerate four-wave mixing signals on either side of the exciting laser spectrum are controlled by amplitude and phase shaping. Quantitative signal modeling for the input pulse parameters shows a spectrally flat phase response of the near-degenerate four-wave mixing due to the linear dispersion of the massless Dirac Fermions
\end{abstract}

\footnotetext{
${ }^{*}$ To whom correspondence should be addressed

${ }^{\dagger 1}$ Department Chemie and CeNS, Ludwig Maximilians Universität München, Butenandtstr. 5-13, 81377 Munich, Germany

$\ddagger_{2}$ Josef Ressel Zentrum, Fachhochschule Vorarlberg, Hochschulstr. 1, 6850 Dornbirn, Austria

${ }^{\top 3}$ Cambridge Graphene Centre, University of Cambridge, 9 JJ Thomson Avenue, Cambridge CB30FA, UK 
in graphene. Exploiting these properties we demonstrate that graphene is uniquely suited for the intra focus phase characterization and compression of broadband laser pulses, circumventing disadvantages of common methods utilizing second or third harmonic light.

Keywords: graphene, four-wave mixing, pulse shaping, femtosecond laser, microscopy, phase retrieval

The linear dispersion of the massless Dirac Fermions in graphene is enabling an ever increasing number of optical and optoelectronic applications. ${ }^{1-4}$ The resulting spectrally flat absorption in combination with ultrahigh electric switching rates make graphene particularly interesting for highspeed applications in photodetectors ${ }^{3,5,6}$ and as broadband saturable absorbers in ultrafast lasers. ${ }^{2,7}$ The linear dispersion is also connected to efficient higher-order optical responses, including nonlinear broadband photoluminescence and four-wave mixing (FWM). ${ }^{8-11}$

Non-degenerate FWM at $\omega_{F W M}=2 \omega_{1}-\omega_{2}$ in graphene was theoretically described in Refs. 9,12 and experimentally demonstrated in Ref. 10. The large non-linearity of graphene's, as shown by a $\chi^{(3)}$ which is 2 orders of magnitude higher than the nonlinearities observed for comparable gold films, ${ }^{13}$ is explained by the fact that all vertical transitions are resonant at all frequencies $\omega_{F W M}, \omega_{1}$ and $\omega_{2} \cdot{ }^{10}$

Ref. 12 theoretically showed that the FWM response is dispersionless with respect to phase and its intensity is spectrally smooth scaling with $\omega^{-4}$. FWM from graphene has been used in or proposed for various applications, such as wavelength conversion in all-fiber configurations, ${ }^{14,15}$ in vitro imaging in biological samples ${ }^{16}$ and phase matching for perfect lens applications. ${ }^{17,18}$

A major challenge in ultrafast microscopy is the dispersion of the optical components and the resulting temporal broadening of laser pulses. This can become a significant problem in the case of high-numerical aperture microscope objectives and other elements such as lenses, windows and dielectric filters. ${ }^{19}$ Common procedures used for intra-focus pulse compression include frequency resolved optical gating (FROG) ${ }^{20}$ and multiphoton intrapulse interference phase scans (MIIPS) ${ }^{21-23}$. These rely on the second harmonic generation from reference materials, e.g. beta barium borate or iron(III) iodate crystals. ${ }^{24}$ These procedures thus require optical components suitable for both 
fundamental and harmonic frequencies. For broadband laser pulses centered from the visible to the near infrared this would require microscope objectives, immersion liquids, substrates and lenses, as well as sensitive detectors that are also suitable for the ultra violett, which are in many cases not available. In particular for epi-detection using the same microscope objective for focusing and collection this is a major difficulty with respect to spectral transmittance and chromatic aberrations.

Here, we propose to use the near-degenerate four-wave mixing signal of graphene as a nonlinear optical signal to determine the spectral phase profile of laser pulses in the focus of microscope objectives. Since near-degenerate FWM occurs at energies similar to the excitation and given the flat spectral response of graphene, this procedure would also be applicable to broadband laser pulses with a temporal duration below $50 \mathrm{fs}$. We find that the emission spectrum of graphene close to the energy of an ultrafast laser pulse is dominated by very intense near-degenerate FWM (ND-FWM). We then show that the spectral phase response of $\chi^{(3)}$ in the range 1.47 to $1.62 \mathrm{eV}$ is flat, implying a dispersionless and instantaneous parametric process for the $15 \mathrm{fs}$ pulses used in our experiment. The non-linear response of graphene is therefore very well suited for phase characterization. We finally demonstrate the compression of a $15 \mathrm{fs}$ laser pulse in the focus of an objective with a numerical aperture (NA) of 1.3.

We study micromechanically exfoliated single layer graphene (SLG) deposited on glass, using confocal scanning microscopy. An oil-immersion objective $(\mathrm{NA}=1.3)$ is used to excite the sample and to collect the back-scattered light. The laser source is a Titanium-Sapphire oscillator, producing trains of $15 \mathrm{fs}$ pulses at a repetition rate of $80 \mathrm{MHz}$. The central photon energy is $1.55 \mathrm{eV}$ (800 $\mathrm{nm})$ and the bandwidth $0.2 \mathrm{eV}(100 \mathrm{~nm})$. We estimate the laser fluence in the focus from the pulse energy of $5.4 \mathrm{pJ}$ and the diameter of the diffraction limited focal spot of $d=0.61 \lambda / N A=375 \mathrm{~nm}$ to be $49 \mathrm{~J} / \mathrm{m}^{2}$. A pulse-shaper in $4 \mathrm{f}$ configuration is used both for compensating the optical dispersion introduced by the setup and for providing additional phase and amplitude modulation. Details about sample preparation and setup are in the Supporting Information.

Fig. 1(a) plots the single layer graphene emission spectrum excited by a 15 fs laser pulse centered at $1.55 \mathrm{eV}$ in the focus of a high $\mathrm{NA}=1.3$ objective. On both sides of the excitation pulse an 
intense signal is seen, decaying rapidly with increasing energy shift. In the following we verify that this signal indeed results from near degenerate four-wave mixing, which is maximized for the shortest (bandwidth limited) pulse.

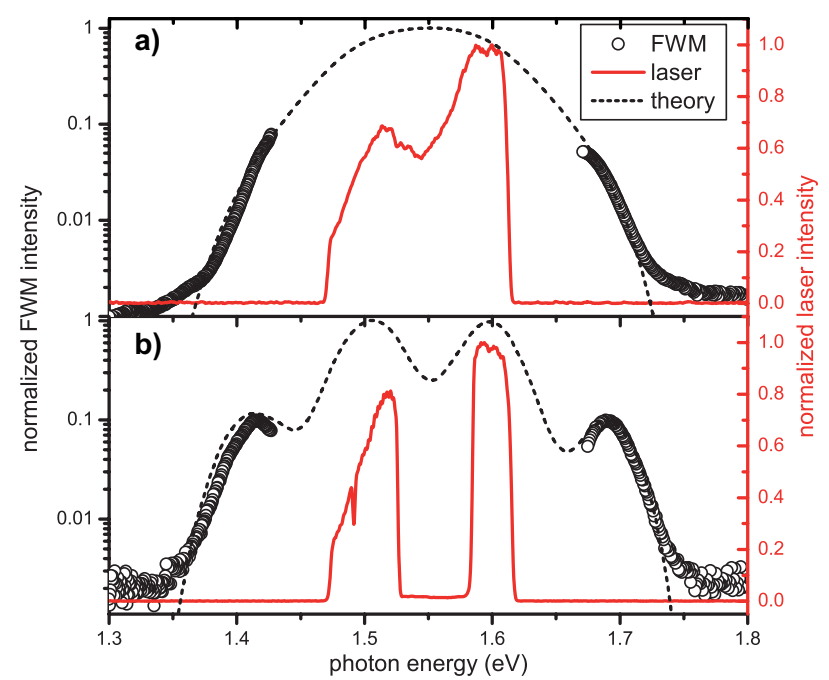

c)
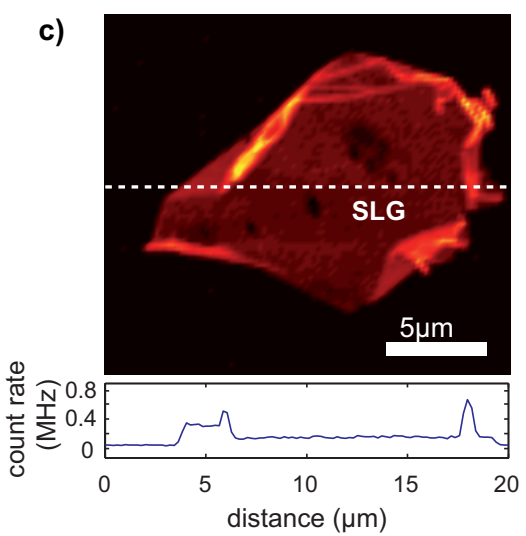

$0 \mathrm{MHz}$

Figure 1: (a) ND-FWM spectrum of single layer graphene (SLG) on glass (semi-log plot). The laser pulse is transform limited in the focus of the objective and has a full width at half maximum of $15 \mathrm{fs}$. The dashed line corresponds to the calculated FWM-spectrum on the basis of a flat spectral amplitude and phase response and the spectrum of the incident laser. (b) The ND-FWM signal originating from a tailored excitation spectrum follows the theoretical prediction. (c) Confocal scan (Stokes side) of SLG on glass. ND-FWM provides a nearly background free signal contrast that highlights features such as wrinkles. The dashed line indicates the position of the cross section.

As a parametric process, four-wave mixing has several realizations fulfilling energy conservation: ${ }^{25} \omega_{F W M}=\left| \pm \omega_{1} \pm \omega_{2} \pm \omega_{3}\right|$. Typically one can distinguish a degenerate and a near-degenerate case, where $\omega_{F W M}^{\text {degenerate }}$ equals one of the three input frequencies and $\omega_{F W M}^{\text {non-degenerate }}$ is different from all of them. ${ }^{10,25}$ In the case of a broadband laser, frequency mixing terms across the spectrum become important and lead to significant contributions directly next to the laser spectrum, defining the near degenerate case. ${ }^{25,26}$

Four-wave mixing spectra can be calculated according to the following integral: ${ }^{25}$

$$
\begin{aligned}
I(\omega)=c \varepsilon_{0} & \mid \chi^{(3)} \int_{\omega_{\min }}^{\omega_{\max }} d \Omega_{1} \int_{\omega_{\min }}^{\omega_{\max }} d \Omega_{2} \quad E\left(\Omega_{1}\right) \cdot E\left(\Omega_{2}\right) \times \\
& \times\left. E\left(\Omega_{1}+\Omega_{2}-\omega\right) \cdot e^{i \cdot\left(\varphi\left(\Omega_{1}\right)+\varphi\left(\Omega_{2}\right)-\varphi\left(\Omega_{1}+\Omega_{2}-\omega\right)\right)}\right|^{2}
\end{aligned}
$$


where $E$ is the amplitude of the excitation laser field, $c$ the speed of light, $\varepsilon_{0}$ the vacuum permittivity, $\omega_{\min }$ and $\omega_{\max }$ are the lower and upper frequency limits of the laser spectrum, and $\varphi(\omega)$ is the spectral phase of the pulse. We determine the total emitted four-wave mixing energy per incoming pulse in two steps. First, we scale the calculated FWM spectrum (dashed line in Fig. 1(a)) to the measured intensity taking into account the overall detection sensitivity of the setup. This allows to access the FWM intensity also in the non-measurable regime covered by the laser pulse. Integrating the scaled spectrum we obtain the full FWM energy per pulse of $10^{-17} \mathrm{~J}$ corresponding to the spectral integral over eq. 1 . From this we determine a value of $\chi^{(3)}=4.3 \times 10^{-6}$ esu for the non-linear susceptibility at a central energy of $1.55 \mathrm{eV}$. This result is in general agreement with the value of $\chi^{(3)}=1.5 \times 10^{-7}$ esu reported in Ref. 10 while the deviation could result from the tight focusing of the excitation pulse in the present measurement in combination with the non-linearity of the signal. 27

According to Refs. 9 and $10, \chi^{(3)}$ in single layer graphene is inversely proportional to the fourth power of the frequency $\left(\chi^{(3)} \propto \omega^{-4}\right)$ but exhibits no phase dependence. Within the $100 \mathrm{~nm}$ spectral bandwidth of our laser pulse this fourth order dependence results in a variation of $40 \%$ at most. Remarkably, the influence of this scaling factor on the emitted intensity is only minor (see Fig. S6 of the supporting material). This is due to the spectral integration and the mixing of the frequency components seen in Fig. 1, which leads to a broad and structureless emission spectrum.

Newer theoretical results ${ }^{28}$ also suggest a general phase dependence of the third order nonlinearity in graphene, but this is negligible in the range studied in this work (see Fig. S7 in the supporting material for details). We thus calculate the ND-FWM spectra using a constant third order susceptibility $\chi^{(3)}$ and the measured laser spectrum (dashed line in Fig. 1(a), (b)). This is in very good agreement with the experimental response, as seen in Fig. 1(a), (b). At energies further away from the excitation, the experimental signal exceeds the theoretical curve, which we attribute to non-linear photoluminescence. ${ }^{8,29,30}$ This contribution is at least two orders of magnitude lower than the four-wave mixing signal. Furthermore, the power dependence is cubic for ND-FWM ${ }^{25}$ and roughly quadratic for non-linear photoluminescence ${ }^{29}$ providing another means to distinguish 
the two contributions.

The case of two spectrally separated excitation pulses $E_{1}(\omega)$ and $E_{2}(\omega)$ is implemented in Fig. 1(b). Here intra-pulse frequency mixing within $E_{1}$ and $E_{2}$ does not contribute to the detected four-wave mixing signal. Only signals from inter-pulse mixing following $\omega_{F W M}^{\text {non-degenerate }}=2 \omega_{1}-$ $\omega_{2}$ are observed with $\omega_{2}>\omega_{1}$, where $\omega_{1}$ and $\omega_{2}$ are the central frequencies of the two pulses. In this case Eq. 1 simplifies to:

$$
I\left(\omega_{S}\right)=c \varepsilon_{0}\left|\chi^{(3)} \int_{\omega_{\min }}^{\omega_{\max }} d \Omega \quad E_{1}^{2}(\Omega) \times E_{2}\left(2 \cdot \Omega-\omega_{S}\right) \cdot e^{i\left(2 \cdot \varphi(\Omega)-\varphi\left(2 \cdot \Omega-\omega_{S}\right)\right)}\right|^{2}
$$

for the Stokes side. We find again a very good agreement between our parameter-free calculation and the experimental spectrum, assuming a spectrally constant $\chi^{(3)}$ response, as shown in Fig. 1(b).

Using either the low or the high energy side of the emission as a signal for an intensity map, we are able to detect high contrast confocal images of graphene as shown in Fig. 1(c). The signal on the detector reaches several million counts per second at a laser pulse energy of $5.4 \mathrm{pJ}$. Therefore it is easily detected. In combination with the weak background stemming probably from the immersion oil and the glass substrate, near degenerate four-wave mixing provides a very clear contrast and high signal-to-noise ratio imaging.

Using amplitude pulse shaping we can readily verify the power dependence of the ND-FWM signal. For the Stokes side, the dependence on the input power is quadratic for $I_{1} \propto\left|E_{1}\right|^{2}$ and linear for $I_{2} \propto\left|E_{2}\right|^{2}$; vice versa for the Anti-Stokes side. The four resulting power laws are confirmed on single layer graphene as shown in Fig. 2(a).

Additionally, it is possible to verify the FWM origin of the signal within the probed range directly by scanning the frequency spacing between the central frequencies of the individual pulses using amplitude shaping (Fig. 2(b)). The dependence of the input frequencies and of the input power as well as the good agreement with the theoretical curves for different spectral pulse shapes confirms that the detected signal stems mainly from FWM with a spectrally flat amplitude $\chi^{(3)}$ response. 
a)

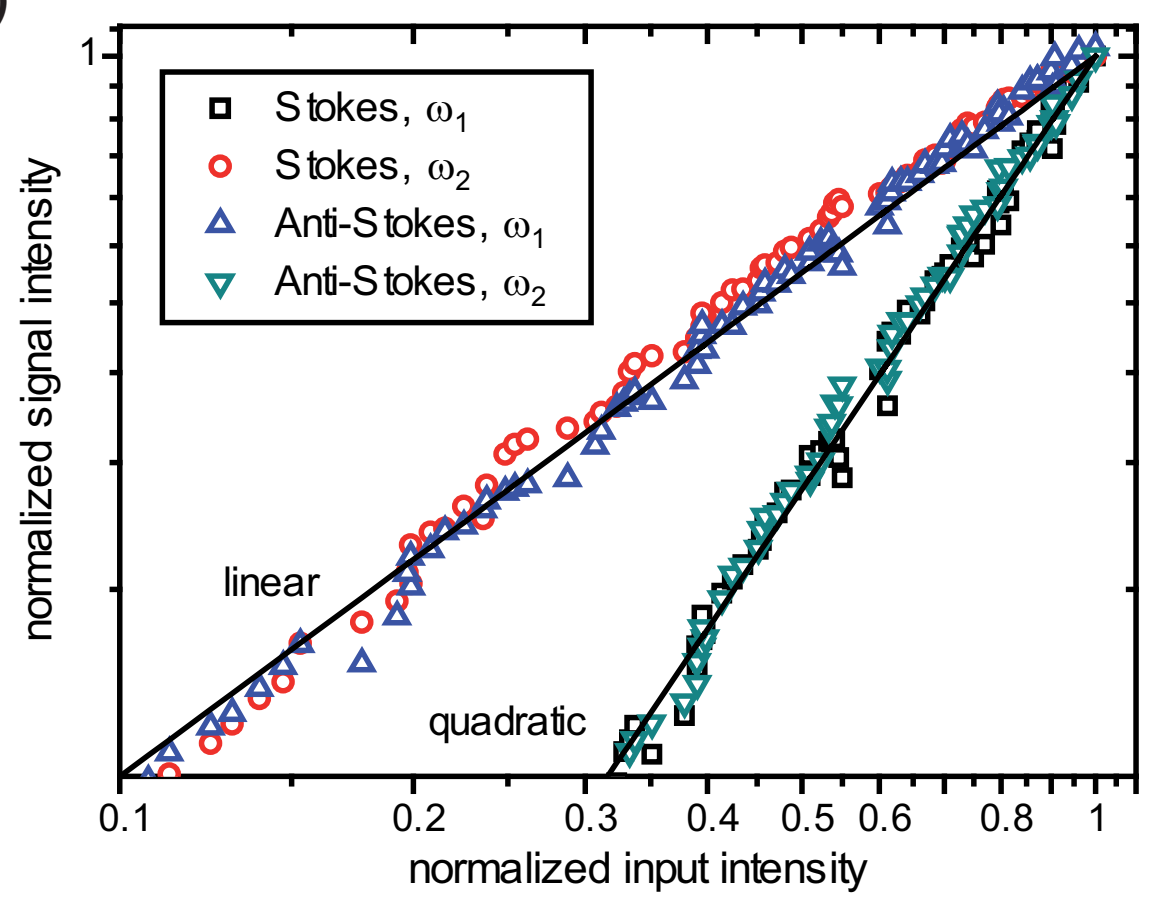

b)

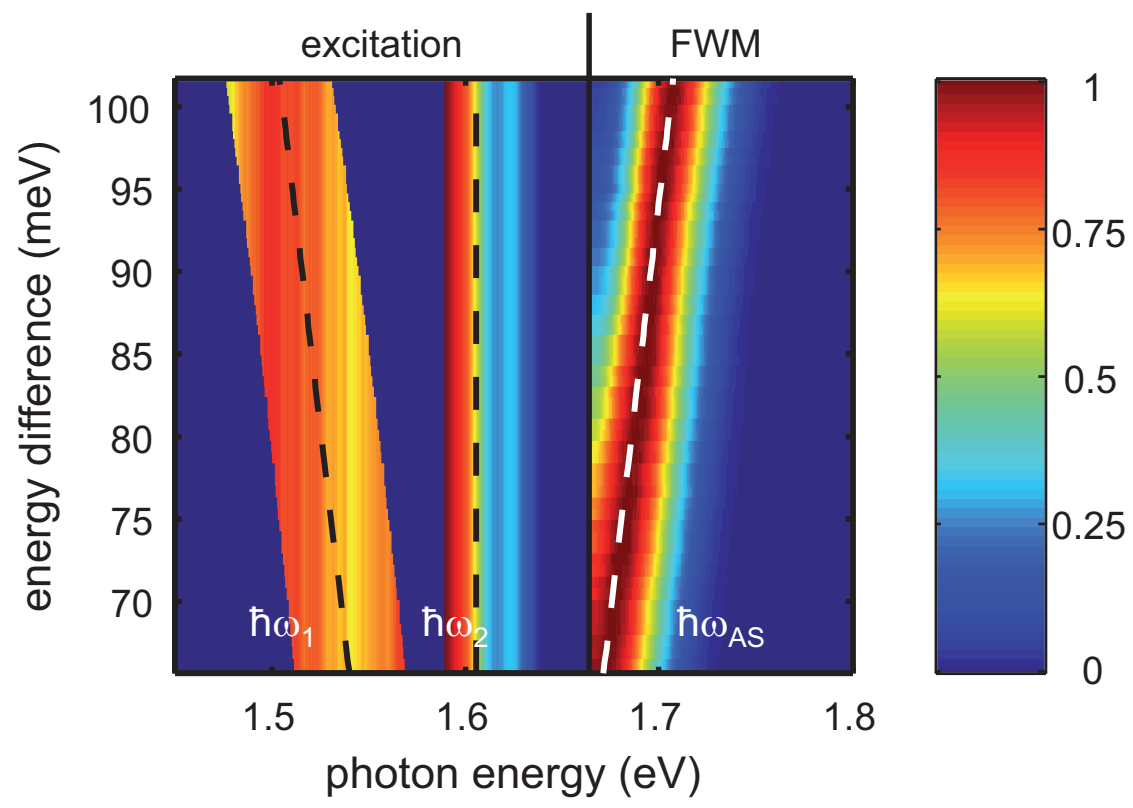

Figure 2: (a) Stokes and Anti-Stokes power dependence of the FWM signal. Two narrow-band pulses are cut out of the full spectrum by the pulse shaper and individually varied in power. The measured power dependencies (symbols) match the theoretical curves (solid lines) in all four possible cases. (b) Control of FWM by scanning the energy difference between two pulses shifts the Anti-Stokes band (Stokes not shown). FWM are normalized to one, $\omega_{1}$ and $\omega_{2}$ are the center-ofmass frequencies of the individual pulses, the Anti-Stokes frequency follows: $\omega_{A S}=2 \omega_{2}-\omega_{1}$. 
a) experiment

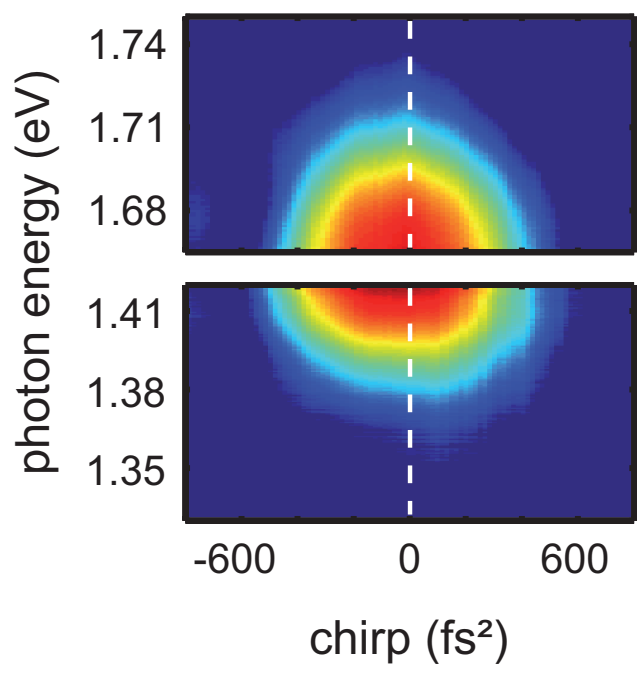

b) simulation

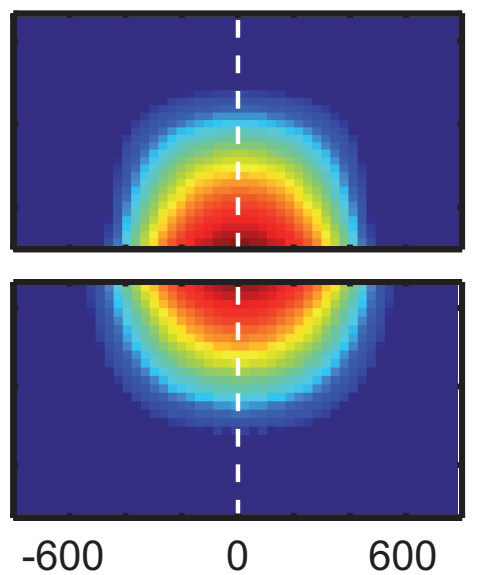

chirp $\left(\mathrm{fs}^{2}\right)$

Figure 3: (a) Normalized SLG-FWM signal as a function of the chirp of the excitation laser pulse (logarithmic scale). The signal maximum is at $0 \mathrm{fs}^{2}$ indicating that the material itself produces the highest signal for a pulse with flat phase (input spectrum as in Fig. 1(a)). (b) Simulation on the basis of a flat phase $\chi^{(3)}$ following eq. 1.

A key requirement for broadband pulse characterization is a well-defined spectral phase response of the sample. In order to check for a phase dependence of the FWM signal in single layer graphene, we perform chirp scans. The chirp $c$ (corresponding to a group delay dispersion) of a laser pulse is the second order polynomial spectral phase: $\varphi(\omega)=\varphi_{0}+\frac{c}{2} \cdot\left(\omega_{0}-\omega\right)^{2} \cdot{ }^{25}$ We apply a well-defined chirp to the pulse using a pulse shaper, and monitor the corresponding ND-FWM signal on the Stokes and Anti-Stokes side (for experimental details see supporting material). Our data shows a strong dependence of the FWM signal on the applied chirp, Fig. 3(a).

This is expected since adding chirp to a femtosecond laser pulse leads to temporal broadening decreasing the maximum field intensity. Importantly, the maximum FWM signal occurs for zero chirp for all photon energies. A shift of the whole pattern along the chirp axis would indicate a second order phase dependence in $\chi^{(3)}$ as follows from Eq.1. The same applies to higher even polynomial orders, while higher odd orders would result in a non-symmetric shape of the pattern. We thus conclude that the chirp dependence is only due to the associated intensity variation and that the graphene phase response causes no additional chirp, i.e. is dispersionless in the probe spectral range. Fig. 3(b) plots the theoretical ND-FWM signal according to Eq. 1. This agrees well 
with the experimental data in Fig. 3(a), corroborating the claim of a flat phase $\chi^{(3)}$.

We now demonstrate that due to its spectrally flat amplitude and phase, the strong near-degenerate four-wave mixing signal from graphene can be used for intra-focus phase characterization and compression of broadband pulses assuming a uniform spatial beam profile. In this scheme, the ND-FWM signal for a laser pulse of unknown phase is maximized by varying the spectral phase profile of the pulse using a pulse shaper. In a first step, the ND-FWM signal is detected while scanning the second and third order of the phase (see Supporting Info Fig. S4). The peak phase is then used as the starting point for the following procedure.

Final reconstruction of the phase, or more precisely its second derivative, is achieved by maximizing the ND-FWM signal using a genetic algorithm. ${ }^{31}$ The second order phase is varied at 9 nodal points over the spectrum, while the parts in between are approximated by cubic interpolation. The number of parameters for the genetic algorithm is 9 , allowing for a rapid convergence, typically within 10 to 20 generations. The actual phase applied to the pulse shaper is calculated by double integration with arbitrary integration constants. This can be done since the first (polynomial) order of the phase does not contribute to the pulse shape and the phase offset (carrier envelope phase) cannot be measured by a simple third order process, while having negligible influence for the shape of a $15 \mathrm{fs}$ laser pulse.

After compressing the pulse by maximizing the ND-FWM signal, the result is compared to the procedure of multiphoton intrapulse interference phase scans (MIIPS ${ }^{21}$ ), using the second harmonic of iron(III) iodate nano-crystals detected in the same microscope. Fig. 4(b) demonstrates only a very small residual phase at the detection limit of the system. An interferometric second harmonic autocorrelation scan further verifies that the pulse is bandwidth limited after compression (Fig. 4(c)). This demonstrates the feasibility of phase characterization using the near-degenerate four-wave mixing signal from single layer graphene.

We now compare our approach with current schemes and materials. From a materials perspective, graphene has several advantages. Its sub-nm thickness provides the optimum focus definition, which is particularly relevant for microscopy applications. In contrast, for other materials such as 
a)

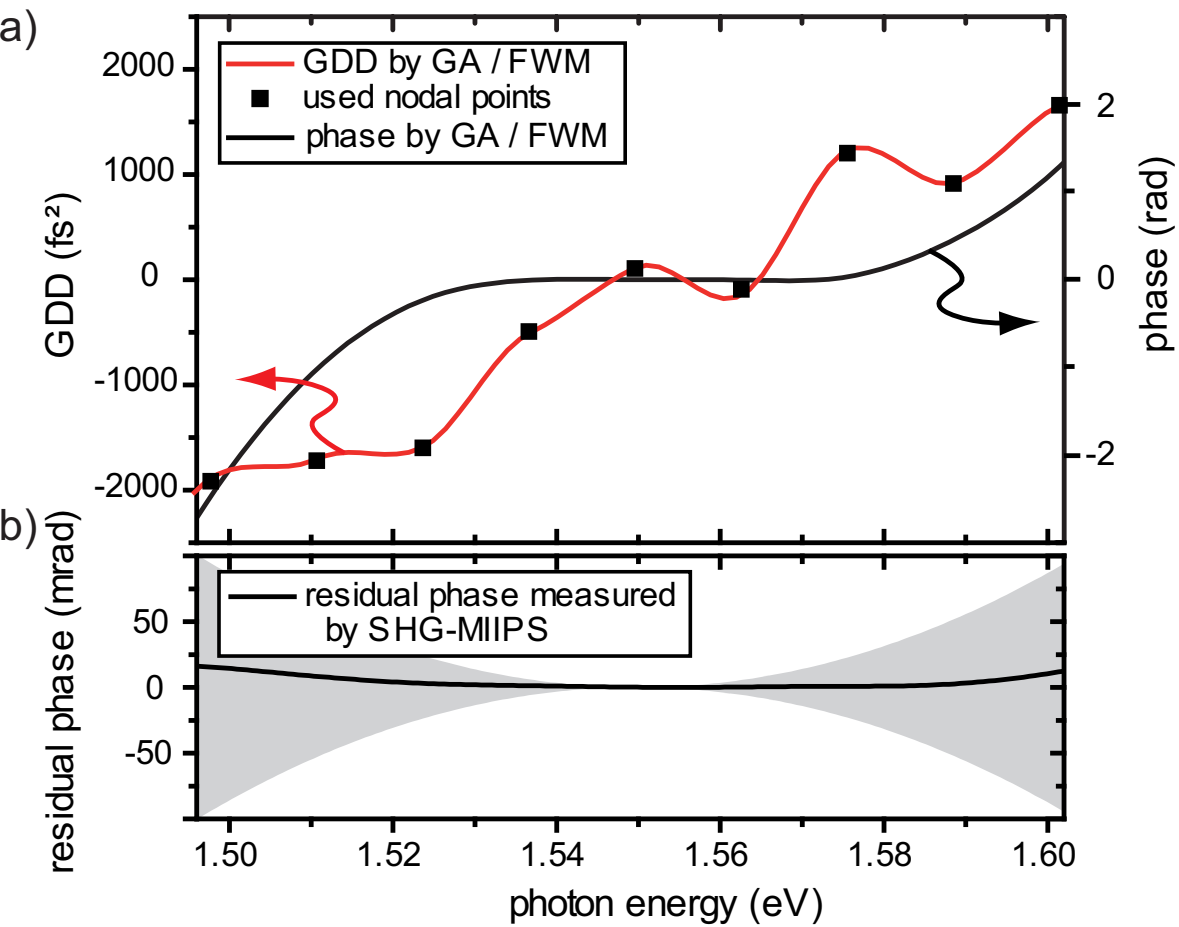

c)

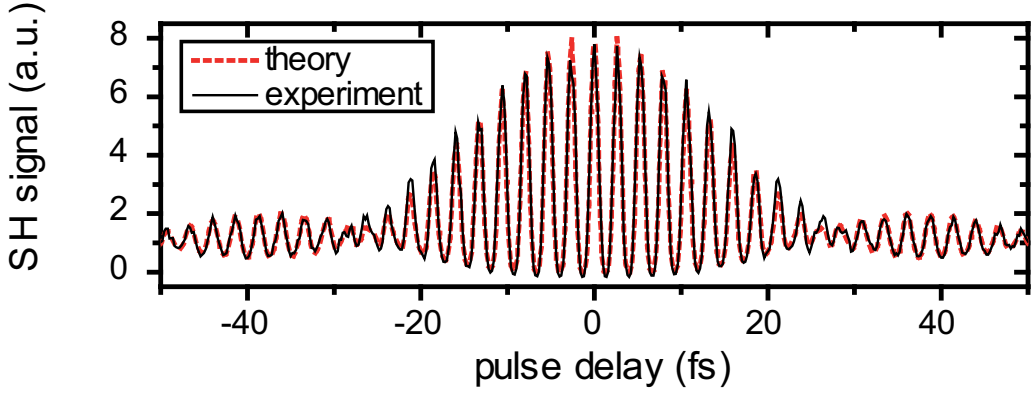

Figure 4: Demonstration of pulse compression using a genetic algorithm (GA) to maximize the ND-FWM signal on the Anti-Stokes side of the laser. (a) Reconstructed group delay dispersion (GDD) of the pulse (red line), approximated by cubic interpolation between 9 nodal points (black symbols), used as free parameters for the optimization. The shown spectral phase (black line) is the double integrated GDD with arbitrary integration constants. (b) Residual phase measured by MIIPS from second harmonic generation (SHG) on iron(III) iodate nanocrystals. The gray area is a conservative estimate on the error. (c) Second harmonic generation auto-correlation with the phase correction of Fig. (a) shows very good agreement with the theoretical curve obtained from the input spectrum.

beta barium borate nanocrystals the size is typically on the order of several tens of nanometers, adding uncertainty to the focal position and depth. In the worst case, this could include propagation effects in the non-linear response, causing erroneous phase corrections. We note that while two-dimensional graphene can be used to define the axial focus position very accurately, it does not provide information on the lateral focus properties. Beam distortions such as a spatial chirp can 
thus not be directly detected. Therefore a clean laser mode is very important for the present measurement. In case of spatially distorted mode profiles, other techniques that are based on nanoscale probes, not directly relying on the mode profile, can be used (see e.g. Refs. 22,32).

At the same time, the graphene four-wave mixing is an extremely efficient process leading to easily detectable signals reaching photon count rates of several millions per second. Most importantly, its spectrally uniform response should allow for phase characterization of laser pulses reaching from the $\mathrm{THz}$ to the visible regime.

Near-degenerate FWM avoids drawbacks of schemes relying on higher harmonic detection and the associated requirements regarding the achromaticity and the large spectral detection range as discussed in the following. This could be particular useful for pulses in the visible and for ultra-broadband pulses. Moreover, near-degenerate FWM at a given detection frequency contains the contribution of a broad range of input frequencies following Eqn.1. The ND-FWM signal is thus maximized if: $\varphi\left(\Omega_{1}\right)+\varphi\left(\Omega_{2}\right)-\varphi\left(\Omega_{1}+\Omega_{2}-\omega\right)=0$ for all combinations of $\Omega_{1}$ and $\Omega_{2}$. This can be achieved for a spectrally flat phase only. Therefore in the limits of $\omega \approx \omega_{\min }$ or $\omega \approx \omega_{\max }$ all spectral components contribute. As a consequence, no spectrometer is required for pulse characterization when using a spectrally integrated signal that ranges sufficiently close to the excitation spectrum. The pulse optimization procedure could therefore be more robust compared to e.g. FROG or MIIPS, which require the detection of the full second harmonic spectrum. ${ }^{20,21}$

We note that for ultra-broadband pulses exceeding $1600 \mathrm{~cm}^{-1}$ bandwidth, non-linear Raman scattering from the $\mathrm{G}$ mode in graphene could contribute to the detected signal. In this case the procedure could be modified, e.g. the spectrum could be compressed step-wise by limiting the bandwidth. We also note that other pulse characterization procedures based on FWM have been presented, e.g. in combination with an additional gate pulse ${ }^{33}$ or spectral detection analogous to FROG. ${ }^{34}$ Compared to these procedures the presented approach based on graphene's neardegenerate four-wave mixing appears to be simpler and more easily implemented. Very recently Tielrooij et al. demonstrated a femtosecond photocurrent response ${ }^{35}$ of graphene which can also be used for pulse measurements based on an electrical readout. 
In summary, we showed that the near-degenerate four-wave mixing signal in single layer graphene can be described by a dispersionless and instantaneous $\chi^{(3)}$-process as expected from the linear band structure. The high and background free signal of several million counts per second makes ND-FWM ideal for non-linear microscopy applications. Since there is no material specific phase influencing the signal, graphene proves to be uniquely suited for phase characterization of ultrafast pulses as demonstrated by compressing a $15 \mathrm{fs}$ pulsed laser. This is of particular advantage for microscopy applications with broadband lasers or lasers in the visible, where second or third harmonic generation as a tool for pulse characterization is no longer feasible because of the large spectral window that has to be covered by the optics.

Supporting Information Available: Experimental details about the pulse shaper, detection scheme, and sample material. This material is available free of charge via the Internet at http: //pubs.acs.org.

\section{Acknowledgments}

We acknowledge support from the Deutsche Forschungsgemeinschaft through SPP 1391 (HA4405/6-

1) and the Nanosystems Initiative Munich (NIM), the ERC starting Grant NEWNANOSPEC (279494), the synergy grant Hetero2D, the EU Graphene Flagship (n. 604391), a Royal Society Wolfson Research Merit Award and the EPSRC grants EP/K01711X/1, EP/K017144/1, EP/L016087/1.

\section{References}

(1) Geim, A. K.; Novoselov, K. S. Nature Materials 2007, 6, 183-191.

(2) Bonaccorso, F.; Sun, Z.; Hasan, T.; Ferrari, A. C. Nature Photon. 2010, 4, 611.

(3) Koppens, F. H. L.; Mueller, T.; Avouris, P.; Ferrari, A. C.; Vitiello, M. S.; Polini, M. Nature Nanotech. 2014, 9, 780 . 
(4) Ferrari, A. C.; Bonaccorso, F.; Falko, V.; Novoselov, K. S.; Roche, S.; Bøggild, P.; Borini, S.; Koppens, F.; Palermo, V.; Pugno, N. Nanoscale 2014,

(5) Xia, F.; Mueller, T.; Lin, Y.-M.; Valdes-Garcia, A.; Avouris, P. Nature Nanotech. 2009, 4, 839-843.

(6) Vicarelli, L.; Vitiello, M. S.; Coquillat, D.; Lombardo, A.; Ferrari, A. C.; Knap, W.; Polini, M.; Pellegrini, V.; Tredicucci, A. Nature Mater. 2012, 11, 865.

(7) Sun, Z.; Hasan, T.; Torrisi, F.; Popa, D.; Privitera, G.; Wang, F.; Bonaccorso, F.; Basko, D. M.; Ferrari, A. C. ACS Nano 2010, 4, 803-810.

(8) Lui, C. H.; Mak, K. F.; Shan, J.; Heinz, T. F. Phys. Rev. Lett. 2010, 105, 127404.

(9) Mikhailov, S. A. EPL 2007, 79, 27002.

(10) Hendry, E.; Hale, P. J.; Moger, J.; Savchenko, A. K.; Mikhailov, S. A. Phys. Rev. Lett. 2010, $105,097401$.

(11) Wu, R.; Zhang, Y.; Yan, S.; Bian, F.; Wang, W.; Bai, X.; Lu, X.; Zhao, J.; Wang, E. Nano Lett. 2011, 11, 5159-5164.

(12) Mikhailov, S.A. Physica E 2012, 44, 924-927.

(13) Xenogiannopoulou, E.; Aloukos, P.; Couris, S.; Kaminska, E.; Piotrowska, A.; Dynowska, E. Opt. Commun. 2007, 275, 217.

(14) Luo, Z.; Zhou, M.; Wu, D.; Ye, C.; Weng, J.; Dong, J.; Xu, H.; Cai, Z.; Chen, L. J. Lightw. Technol. 2011, 29, 2732-2739.

(15) Xu, B.; Martinez, A.; Yamashita, S. IEEE Photon. Technol. Lett. 2012, 24, 1792-1794.

(16) Li, B.; Cheng, Y.; Liu, J.; Yi, C.; Brown, A. S.; Yuan, H.; Vo-Dinh, T.; Fischer, M. C.; Warren, W. S. Nano Letters 2012, 12, 5936-5940. 
(17) Pendry, J. B. Science 2008, 322, 71-73.

(18) Harutyunyan, H.; Beams, R.; Novotny, L. Nature Physics 2013, 9, 423-425.

(19) Lozovoy, V. V.; Dantus, A. M. Chem. Phys. Chem. 2005, 6, 1970.

(20) Trebino, R.; DeLong, K. W.; Fittinghoff, D. N.; Sweetser, J. N.; Krumbügel, M. A.; Richman, B. A.; Kane, D. J. Rev. Scie. Instr. 1997, 68, 3277-3295.

(21) Lozovoy, V. V.; Pastirk, I.; Dantus, M. Optics Letters 2004, 29, 775-777.

(22) Accanto, N.; Nieder, J.-B.; Piatkowski, L.; Castro-Lopez, M.;Francesco, P.; Brinks, D.;van Hulst, N. Light Sci. Appl. 2014, 3, e143.

(23) Comin, A.; Ciesielski, R.; Piredda, G.; Donkers, K.; Hartschuh, A. J. Opt. Soc. Am. B 2014, $31,1118-1125$.

(24) Bonacina, L.; Mugnier, Y.; Courvoisier, F.; Dantec, R. L.; Extermann, J.; Lambert, Y.; Boutou, V.; Galez, C.; Wolf, J. Appl. Phys. B 2007, 87, 399.

(25) Boyd, R. W. Nonlinear Optics; Academic Press, 3rd ed., 2003.

(26) Min, W.; Lu, S.; Rueckel, M.; Holtom, G. R.; Xie, X. S. Nano Lett. 2009, 9, 2423.

(27) Due to the tight focusing of fields and the resulting spatially non-uniform intensity non-linear material constants are difficult to quantify in microscopic measurements. The reported value for $\chi^{(3)}$ represents the equivalent obtained for plane wave excitation with a circular diameter of $375 \mathrm{~nm}$. While the individual characteristics of the components in the detection beam path are well known, such as filter transmission and camera sensitivity, they add up to a total uncertainty of about an order of magnitude .

(28) Mikhailov, S. A. Phys. Rev. B 2014, 90, 241301.

(29) Stöhr, R. J.; Kolesov, R.; Pflaum, J.; Wrachtrup, J. Phys. Rev. B 2010, 82, 121408. 
(30) Winzer, T.; Ciesielski, R.; Handloser, M.; Comin, A.; Hartschuh, A.; Malic, E. Nano Lett. 2015, 15, 1141-1145.

(31) Mathworks Inc., Optimization Toolbox. 2009.

(32) Pawłowska, M.; Goetz, S.; Dreher, C.; Wurdack, M.; Krauss, E.; Razinskas, G.; Geisler, P.; Hecht, B.; Brixner, T. Opt. Express 2014, 22, 31496.

(33) Selm, R.; Krauss, G.; Leitenstorfer, A.; Zumbusch, A. Opt. Express 2012, 20, 5955.

(34) Petrillo, K. G.; Wang, K.-Y.; Foster, A. C.; Foster, M. A. Opt. Express 2013, 21, 31229.

(35) Tielrooij, K. J.; Piatkowski, L.; Massicotte, M.; Woessner, A.; Ma, Q.; Lee, Y.; Myhro, K. S.; Lau, C. N.; Jarillo-Herrero, P.; van Hulst, N.; Koppens, F. H. L. Nat. Nanotech. 2015, doi:10.1038/nnano.2015.54. 


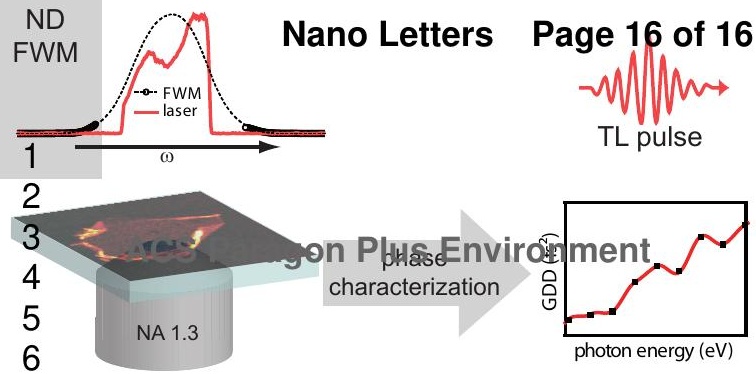

